\title{
Reflecting on design principles in the context of virtual reality learning experiences in astronomy and science education
}

\author{
Magdalena Kersting2,*, Jackie Bondell and Rolf Steier
}

Citation: Lastname, F.; Lastname, F.; Lastname, F. Title. Universe 2021, 7, x. https://doi.org/10.3390/xxxxx

Academic Editor: Firstname Lastname

Received: date

Accepted: date

Published: date

Publisher's Note: MDPI stays neutral with regard to jurisdictional claims in published maps and institutional affiliations.

Copyright: (c) 2021 by the authors. Submitted for possible open access publication under the terms and conditions of the Creative Commons Attribution (CC BY) license (http://creativecommons.org/licenses /by/4.0/).

\begin{abstract}
Virtual reality (VR) technologies have the potential to profoundly transform learning activities in astronomy and science through new forms of perceptual engagement and bodily participation. Since advancements in technology and education drive change in each other, researchers have started to explore opportunities and challenges of using VR in (in)formal learning environments. While research on VR from technology perspectives is extensive, there are relatively few attempts to explore learning-oriented design considerations with VR tools.
\end{abstract}

This study is grounded in the education and public-outreach program of the Australian Research Council Centre of Excellence for Gravitational Wave Discovery (OzGrav). The OzGrav-team is an interdisciplinary team of science educators, VR developers, digital artists and astrophysicists that have developed VR outreach programs and school incursions in astronomy.

The goal of this study is to identify the experiential affordances of VR in informal learning contexts and to reflect on potential design principles for VR learning experiences in astronomy and science. To articulate issues related to design and learning with VR from multiple perspectives, we unpack critical design decisions of the OzGrav VR learning resources in the form of a dialogue between VRpractitioners and educational researchers. The dialogue draws on primary data from focus-groupinterviews with the OzGrav-team. We supplement the dialogue with audio-visual material of a VR experience about the virtual universe. Our findings centre on aspects of

1. collaboration and social interaction in VR-learning,

2. negotiating roles while being an observer/participant in the VR-experience,

3. embodiment in an immersive VR environment,

4. finding a balance between visual richness and accessibility of VR-experiences,

5. limitations of what VR environments can visualize.

As the use of VR learning environments gains momentums, our findings contribute to a deeper understanding of the new learning contexts that VR technology can create in astronomy and modern science education.

Keywords: physics education; astronomy education; virtual reality; design principles 